\title{
(2) OPEN ACCESS \\ Sleep problems and risk of all-cause cognitive decline or dementia: an updated systematic review and meta-analysis
}

\author{
Wei Xu ๑1 , ${ }^{1}$ Chen-Chen Tan, ${ }^{1}$ Juan-Juan Zou, ${ }^{2}$ Xi-Peng Cao, ${ }^{3}$ Lan $\operatorname{Tan}^{1}$
}

- Additional material is

published online only. To view please visit the journal online (http://dx.doi.org/10.1136/ innp-2019-321896)

${ }^{1}$ Neurology Department, Qingdao Municipal Hospital Group, Qingdao University, Qingdao, Shandong, China

2Department of

Otorhinolaryngology, Qilu

Hospital of Shandong University; NHC Key Laboratory of

Otorhinolaryngology, Shandong University, Jinan, China

${ }^{3}$ Clinical Research Center, Qingdao Municipal Hospital Group, Qingdao, Shandong, China

Correspondence to DrWei Xu, Neurology department, Qingdao Municipal Hospital Group, Qingdao, Shandong, China; 1037219730@qq.com

Received 20 August 2019 Revised 28 October 2019 Accepted 11 December 2019 Published Online First 26 December 2019

Check for updates

(C) Author(s) (or their employer(s)) 2020. Re-use permitted under CC BY-NC. No commercial re-use. See rights and permissions. Published by BMJ.

To cite: Xu W, Tan C-C, Zou J-J, et al. J Neurol Neurosurg Psychiatry 2020:91:236-244.

\section{ABSTRACT \\ Objectives To conduct an updated systematic review and meta-analysis of association between sleep and all- cause cognitive disorders.}

Methods PubMed and EMBASE were searched from inception to 18 February 2019. Cohort studies exploring longitudinal associations of sleep with cognitive decline or dementia were included. The multivariable-adjusted effect estimates were pooled by random-effects models, with credibility assessment. The robusterror meta-regression model was used to conduct the doseresponse meta-analysis for sleep duration.

Results 11155 reports were searched and 51 eligible cohorts with 15 sleep problems were included for our meta-analyses. Ten types of sleep conditions or parameters, including six (insomnia, fragmentation, daytime dysfunction, prolonged latency, rapid eye movement sleep behaviour disorder and excessive time in bed) with moderate-to-high levels of evidence, were linked to higher risk of all-cause cognitive disorders. Furthermore, a U-shaped relationship was revealed for the associations with sleep duration.

Conclusions Sleep management might serve as a promising target for dementia prevention.

\section{INTRODUCTION}

Lines of evidence showed that sleep disorders could contribute to cognitive decline or dementia (cognitive disorders) and might serve as a promising target for dementia prevention. Evidence suggested sleep could influence core biomarkers of Alzheimer's disease (AD). Difficulty in falling asleep, ${ }^{1}$ poor sleep quality, ${ }^{2}$ sleep loss, ${ }^{3}$ excessive daytime sleepiness ${ }^{4}$ and sleep disordered breathing ${ }^{5}$ were suggested to increase cerebral $\mathrm{A} \beta$ deposition in non-demented elderly. Apnoea $^{6}$ or obstructive sleep apnoea syndrome $^{7}$ was related to higher levels of AD-related neuronal injury biomarkers (ie, P-Tau and T-Tau). Sleep quality could even modulate the protective effects of other environmental factors such as physical exercise ${ }^{8}$ on brain $\mathrm{A} \beta$ deposition. Furthermore, prospective cohort studies have found that various sleep conditions or parameters, such as insomnia, obstructive sleep apnoea, ${ }^{10}$ sleep-related behaviours disorder, ${ }^{11}$ and changed sleep duration, ${ }^{12}$ could significantly elevate the risk of cognitive disorders among non-demented adults. However, the robustness of the evidence base might be jeopardised by sources of bias, ${ }^{13}$ such as high heterogeneity (due to for example, population characteristics, varying definitions of outcome and sleep-related exposures), recall bias and small-sample effect. In the past 2 years, large amounts of cohort studies have sprung up to explore the longitudinal influences of sleep-related exposures on incident risks of cognitive disorders, which necessitates an updated systematic review and meta-analysis.

Herein, we meta-analysed the associations between sleep (including insomnia and its components, sleep-related problems, sleep duration and change of sleep pattern) and all-cause cognitive disorders based on longitudinal cohort studies. Evidence ratings were performed according to risk of bias, inconsistency and imprecision.

\section{METHODS}

Search strategy and selection criteria

We followed the recommendations by the Preferred Reporting Items for Systematic Reviews and Meta-Analyses 2009 guidelines. ${ }^{14}{ }^{15}$ PubMed and EMBASE were searched using the strategy: (((()((dementia) OR Alzheimer) OR cognition) OR cognitive $))$ AND $((((((($ sleep) OR insomnia) OR sleepiness) OR somnolence) OR snoring) OR restless legs syndrome) OR periodic limb movement disorders) OR parasomnias))) AND risk, till 18 February 2019. Bibliographies of relevant original studies and systematic reviews were hand-searched in case of omission. The inclusion criteria were as follows: (i) the study used a longitudinal design that requires at least two measurements of cognition during follow-up, (ii) the study explored the association of sleep conditions or parameters with risk of dementia or cognitive decline and (iii) the study provided the risk estimates or the raw data that can be used to calculate these numbers. No restriction was applied on language. Studies were excluded if they met any of the following criteria: (1) risk estimate is not accessible, (2) cross-sectional studies, (3) only abstracts were available and (4) editorials or comments. Literature selection was performed by two experienced investigators (WX and C-CT) and any disagreements on inclusion were resolved by consensus and arbitration within the review team (WX, C-CT and LT).

\section{Data extraction}

Predesigned templates were used to extract the data, including first author, publication year, study design, cohort name, country, cognitive status at baseline, follow-up duration, attrition rate during 
follow-up, total sample size and incident case number for analysis, mean age, female percentage, outcome definition, type and measurement of sleep disturbance, adjusted confounders and the multivariable-adjusted risk estimates. If any data mentioned above were unavailable, we attempted to obtain them via contacting the corresponding authors. The data extraction was performed by two experienced investigators (WX and C-CT) and any discrepancies were addressed by negotiation with the third reviewer (LT).

\section{Assessment of the study quality and credibility of meta- analyses}

An evolving Newcastle-Ottawa Quality Assessment Scale (NOS) for observational cohort studies was employed to evaluate the quality of eligible studies. The total score of NOS was regarded here as a proxy to assess the overall risk of bias for each single study. The score for each item evaluated the associated risk of bias (online supplementary appendix 1). The credibility of each meta-analysis result was then categorised into four levels: 'Good (G level)', 'Acceptable (A+/-level)', 'Susceptible (S+/-level)' and 'Poor (P level)' according to a combined score of three domains: risk of bias, inconsistency and imprecision (online supplementary appendix 2). In particular, $\mathrm{G}$ and $\mathrm{A}+$ levels were regarded as moderate-to-high credibility. Publication bias was detected but not considered in evidence rating, because (1) not all metaanalyses were suitable for test of publication bias (number of included studies $(\mathrm{N})<10$ ) and $(2)$ for those eligible, we found no evidence of publication bias (see the Results section).

\section{Statistical analyses}

The multivariable-adjusted risk estimates and 95\% CI were logtransformed and pooled using random models (DerSimonianLaird method). ${ }^{16}$ Some studies reported odd ratios (ORs) but not relative risks (RRs) or HRs. Given that ORs tend to overestimate the effect sizes compared with RRs/HRs particularly when the incidence is not low, we transformed ORs to RRs using the following algorithm: ${ }^{17}$

$$
\mathrm{RR}_{\text {adjusted }}=\mathrm{OR}_{\text {adjusted }} /\left[\left(1-\mathrm{P}_{0}\right)+\left(\mathrm{P}_{0} \times \mathrm{OR}_{\text {adjusted }}\right]\right.
$$

$\mathrm{P}_{0}$ indicates the incidence of endpoint (dementia or cognitive decline) in the non-exposed group of cohort. When $\mathrm{P}_{0}$ is not available, the incidence rate of total sample was used as a proxy. ${ }^{17}$ A $95 \%$ prediction interval was calculated to better evaluate the precision of the result. ${ }^{18}$ Heterogeneity was assessed by $\mathrm{Q}$ test and quantified by the $\mathrm{I}^{2}$ metric. The source of heterogeneity was explored via sensitivity analyses, metaregression (if $\mathrm{n} \geq 10$ ) and subgroup analyses according to multiple variables, including study design, region, gender, sample size, cognitive status at baseline, age stage (midlife vs late-life), follow-up duration, adjusted confounder (hypnotics, APOE4 and depression), outcome, exposure definition, effect estimate and quality score. The robustness of the results was examined by excluding those rated as at a higher risk of bias. Publication bias was assessed (if $n \geq 10$ ) following two steps: (1) testing the symmetry of the funnel plot by Egger method and (2) determining whether any asymmetry was due to publication bias via enhanced-contour funnel plots after the trim-and-fill method.

Taking into account the following cases wherein results might be biassed, multiple subgroup and sensitivity analyses were conducted. First, some studies recruited people without dementia at baseline and others specifically constrained the population to those with unimpaired cognition. Notably, inclusion of individuals with mild cognitive impairment, who might be at prodromal stage of $\mathrm{AD}$, resulted in a degree of misclassification bias, especially when the population was in their advanced age and was followed insufficiently. Thus, subgroup analyses according to the cognitive status at baseline, follow-up sufficiency (Q7 of NOS) and life-stage were performed. Moreover, sensitivity analyses excluding studies with poor generalisability (Q1), inadequate follow-up (Q7) and high attrition rates (Q8) were conducted (Appendix 1). The 'metagen', 'metabias' and 'trimfill' packages in R V.3.4.3 software (https://www.r-project.org) were used to perform all these analyses.

A U-shape relationship was indicated for the association between dementia and sleep duration. In the present study, we first summarised the risk estimates based on the comparison of the extreme categories (highest vs middle level and lowest vs middle level). Separate analyses according to outcome (dementia or cognitive decline, $\mathrm{AD}$ and $\mathrm{VD}$ ) and exposure (nocturnal and total daily duration including daytime naps) were performed. Next, we examined the exposure-response relationship between sleep duration and cognitive disorders for non-linearity by fitting a restricted cubic spline model. We used the inverse variance weighted least squares regression with cluster robust error variances (REMR model). ${ }^{19}{ }^{20}$ For studies wherein the reference group was not the lowest category (eg, exposure was defined as tertiles and the middle one is set as the reference group), we regraded the lowest category as the reference and recalculate the effect size using the method by Orsini. ${ }^{21}$ We assigned the midpoint of the upper and lower boundaries in each category of sleep duration as the average level. For studies with an open-ended boundary; we multiplied or divided the reported boundary by 1.25 . Stata V.12.0 was used to conduct the doseresponse analyses.

\section{RESULTS}

\section{Searching results}

Figure 1A exhibits the flow diagrams of the study selection process. The search yielded 11155 articles after deduplication. After scanning the titles and abstracts, 72 articles were considered as potentially eligible. After reviewing the full-texts, we further excluded 16 literatures for varied reasons (figure 1A). After further integrating with additional four papers from the bibliography, a total of 51 cohorts in North America (43\%), Europe (37\%) and East Asia (20\%) were finally included (online supplementary appendix 3$)$.

\section{Characteristics of studies}

The detailed characteristics of studies included in the metaanalysis are shown in table 1 . Most studies reported the association of sleep with dementia (61\%), $\mathrm{AD}(39 \%)$ or cognitive decline (41\%) and only a few involved VD (16\%) (figure 1B). In these studies, we found 15 types of sleep-related exposures eligible for meta-analyses, including insomnia, inadequate/overlong sleep duration, sleep behavioural disturbance (SBD), apnoea, long time in bed (TIB), snoring, napping, and change in sleep pattern and seven insomnia components (including daytime dysfunction, efficiency, fragmentation, adequacy, frequency, latency and subjective quality) (figure 1C).

\section{Insomnia and cognitive disorders}

Insomnia was significantly associated with $27 \%$ higher risk of cognitive disorders $\left(\mathrm{RR}=1.27,95 \% \mathrm{CI}=1.16\right.$ to $\left.1.39, \mathrm{I}^{2}=82 \%\right)$ after pooling findings of 23 cohort studies (260915 participants and 30027 incident cases). Meta-regression revealed that no factors can explain the source of heterogeneity. Sensitivity analysis excluding three studies ${ }^{22-24}$ will lower $\mathrm{I}^{2}<40 \%$ without 


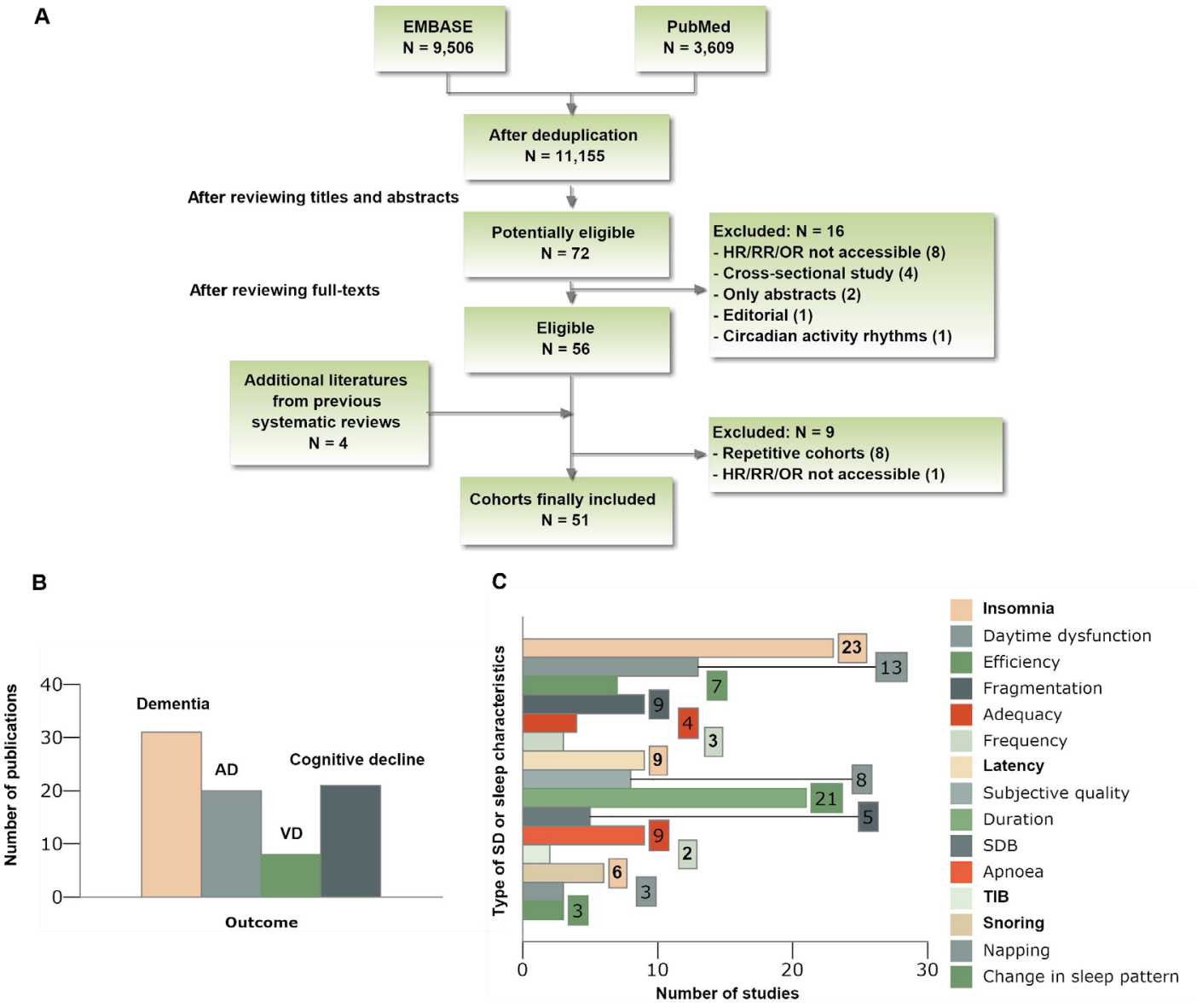

Figure 1 Search flowchart and summary characteristics of included studies. The search yielded 11155 literatures after deduplication. After the standardised literature selection, a total of 51 literatures on 41 cohorts in North America (43\%), Europe (37\%) and East Asia (20\%) were finally included (A). Most studies reported the association of sleep problems with dementia (61\%), AD (39\%) or cognitive decline (41\%) and only a few involved VD (16\%) (B). In these studies, we found eight types of sleep-related problems and seven insomnia-related characteristics for meta-analyses (C). AD, Alzheimer's disease; $\mathrm{OR}$, odds ratio; $\mathrm{RR}$, relative risk; $T \mathrm{IB}$, time in bed.

influence on the significance of the primary result (RR changed from $1.27 \%$ to 1.19 . $95 \% \mathrm{CI}=1.12$ to 1.25$)$. Subgroup analysis indicated that the significance of the primary result was not altered by study design, region, gender, cognitive status at baseline, age stage, effect estimate, quality score or $\mathrm{AD}$ as an outcome (figure 2). However, the pooled results tend to be non-significant in strata of small-sample studies, studies with longer follow-up, studies adjusting for more covariates, studies with VD as outcome and insomnia defined as continuous variable (figure 2). The conclusions seemed more homogeneous in middle-aged population than late-life elderly. No publication bias was revealed for primary $(\mathrm{p}=0.183)$ or subgroup analyses.

\section{Components of sleepdisorder and cognitive disorders}

The high heterogeneity observed above might be explained by heterogeneous definitions of sleepdisorder (online supplementary appendix 3 ). To validate this hypothesis, we conducted separate meta-analyses according to different components. Among seven components for meta-analyses, four were associated with 7\%-16\% increased risk of cognitive disorders, including daytime dysfunction $\left(\mathrm{RR}=1.16,95 \% \mathrm{CI}=1.06\right.$ to $\left.1.27, \mathrm{I}^{2}=38 \%\right)$, inefficiency ( $R R=1.15,95 \% \mathrm{CI}=1.02$ to $\left.1.30, \mathrm{I}^{2}=83 \%\right)$, fragmentation $\left(\mathrm{RR}=1.11,95 \% \mathrm{CI}=1.05\right.$ to $\left.1.17, \mathrm{I}^{2}=0 \%\right)$ and latency $\left(\mathrm{RR}=1.07,95 \% \mathrm{CI}=1.00\right.$ to $\left.1.15, \mathrm{I}^{2}=26 \%\right)$. Adequacy, high frequency of insomnia, and subjective quality showed no significant associations in primary analyses (figure 3 ).
Despite low heterogeneity, meta-regression revealed that sample size $(p<0.05$ for latency) and exposure definition $(\mathrm{p}<0.005$ for daytime dysfunction) could fully explain the source of heterogeneity. In subgroup analyses, inadequacy was associated with $25 \%$ higher risk of dementia $(R R=1.25$, $95 \% \mathrm{CI}=1.04$ to $1.44, \mathrm{I}^{2}=18 \%$ ) and inefficiency could elevate the risk of cognitive decline by $24 \%$ (95\% CI=1.03 to 1.50 , $\mathrm{I}^{2}=31 \%$ ) when it was defined categorically (online supplementary appendix 4).

\section{Sleep-related problems and cognitive disorders}

We also identified four other types of sleep-related problems that showed significant associations with risk of cognitive disorders, including rapid eye movement sleep behavioural disorder (RBD, $\mathrm{RR}=1.90,95 \% \mathrm{CI}=1.23$ to $\left.2.91, \mathrm{I}^{2}=0 \%\right)$, apnoea $(\mathrm{RR}=1.29$, $95 \% \mathrm{CI}=1.12$ to $\left.1.48, \mathrm{I}^{2}=40 \%\right)$, long $\mathrm{TIB} \quad(\mathrm{RR}=1.15$, $95 \% \mathrm{CI}=1.02$ to $1.30, \mathrm{I}^{2}=22 \%$ ) and habitual napping (high trend: $\mathrm{RR}=0.46,95 \% \mathrm{CI}=0.21$ to $\left.1.01, \mathrm{I}^{2}=45 \%\right)$. We identified no significant association with restless leg syndrome (RLS) or snoring (figure 4). Subgroup or sensitivity analyses did not change the above results.

\section{Sleep duration and cognitive disorders}

A total of 21 prospective cohort studies reported the association between sleep duration and cognitive disorders. Three 
Table 1 Characteristics of 51 studies included in the meta-analysis

\begin{tabular}{|c|c|c|c|c|c|c|}
\hline First author, year * & $\begin{array}{l}\text { Design, cohort name, } \\
\text { country }\end{array}$ & Observation & Sample for analysis/cases & Mean age; female & Outcome & $\begin{array}{l}\text { Type of sleep } \\
\text { disorders }\end{array}$ \\
\hline Suh, 2018 & South Korea & $4 y(\max )$ & $2,238 / 265$ & $68.1 ; 54 \%$ & Cognitive decline & Sleep characteristics \\
\hline \multirow[t]{4}{*}{ Sindi, 2018} & Sweden & $9 y(\max )$ & $437 / 19$ & $70 ; 60 \%$ & \multirow[t]{4}{*}{ Dementia } & \multirow{4}{*}{$\begin{array}{l}\text { Insomnia and sleep } \\
\text { duration }\end{array}$} \\
\hline & Sweden & $9 y(\max )$ & $306 / 122$ & $83.9 ; 85 \%$ & & \\
\hline & \multirow[t]{2}{*}{ Finland } & 21y (mean); 26y (max) & $1,409 / 61$ & $50.2 ; 62 \%$ & & \\
\hline & & $10 y$ (mean) & $703 / 44$ & $70.2 ; 65 \%$ & & \\
\hline Ohara, 2018 & Japan & $8.8 y$ (mean); $10 y$ (max) & $1,497 / 294$ & $70 ; 56 \%$ & Dementia & Sleep duration \\
\hline Nakakubo, 2018 & Japan & $4 y(\max )$ & $2,096 / 280$ & $71 ; 53 \%$ & Cognitive decline & $\begin{array}{l}\text { Sleep duration; } \\
\text { excessive daytime } \\
\text { sleepiness }\end{array}$ \\
\hline Lysen, 2018 & Netherlands & $8.5 y$ (mean) & $4,835 / 420$ dementia (320 AD) & $71.9 ; 58 \%$ & Dementia; AD & $\begin{array}{l}\text { Sleep quality; PSQI } \\
\text { components }\end{array}$ \\
\hline Lutsey, 2018 & USA & $\begin{array}{l}14.9 y \text { (median); } 17.5 y \\
(\max )\end{array}$ & $\begin{array}{l}1,083 \text { for apnoea; } 1,653 \text { for } \\
\text { sleep duration/145 }\end{array}$ & $62.7 ; 53 \%$ & Dementia & Apnoea; sleep duration \\
\hline Lu, 2018 & Japan & $5.7 y$ (mean) & $7,422 / 688$ & $74.7 ; 56 \%$ & Dementia & Sleep duration \\
\hline \multirow[t]{2}{*}{ Li, 2018} & \multirow[t]{2}{*}{ USA } & $11 y$ (max); 4.6y (mean) & $1,097 / 220$ & $81.0 ; 77 \%$ & $A D$ & \multirow[t]{2}{*}{ Sleep quality } \\
\hline & & $11 y$ (max); 3.4 (mean) & $855 / 344$ & $80.1 ; 79 \%$ & $\mathrm{MCl}$ & \\
\hline Li, 2018 & USA & $\max >30 y$ & $2,461 / 227$ & $51 ; 50 \%$ & Dementia & Sleep duration \\
\hline Larsson, 2018 & Sweden & $12.6 y$ (mean) & $28,775 / 3,755$ & $71.6 ; 47 \%$ & $A D$ & Sleep duration \\
\hline Burke, 2018 & USA & $4.24 y$ (mean) & $9,184 / 361$ & $71 ; 65 \%$ & Probable AD & Sleep disturbance \\
\hline Westwood, 2017 & USA & $10 y(\max )$ & 2,457/234 dementia (181 AD) & $72 ; 57 \%$ & Dementia; AD & Sleep duration \\
\hline Sung, 2017 & China-Taiwan & $10 y(\max )$ & $184,158 / 5,301$ & $49 ; 65 \%$ & Dementia & $\begin{array}{l}\text { Nonapnoea sleep } \\
\text { disorders }\end{array}$ \\
\hline Chang, 2013 & China-Taiwan & 4.78 to $4.93 y$ (mean) & $8,484 / 199$ & Mixed; $41 \%$ & Dementia & Sleep apnoea \\
\hline Reijs, 2017 & Finland & $2.4 y$ (mean) & $353 / n a$ & $70.6 ; 59 \%$ & $A D$ & Sleep problem \\
\hline Pase, 2017 & USA & $12 y(\operatorname{mean}) ; 19 y(\max )$ & $321 / 32$ dementia (24 AD) & $67 ; 50 \%$ & Dementia; AD & $\begin{array}{l}\text { Percentage of REM } \\
\text { sleep }\end{array}$ \\
\hline Luojus, 2017 & Finland & $21.9 y$ (mean) & $2,386 / 287$ dementia (234 AD) & $53 ; 0 \%$ & Dementia; AD & Sleep disturbance \\
\hline Gabelle, 2017 & France & $1 y(\max )$ & $479 / 63$ & $74 ; 68 \%$ & Cognitive decline & Sleep characteristics \\
\hline Bokenberger, 2017 & Sweden & $\begin{array}{l}17.7 y(\max ) ; 14.3 y \\
\text { (median) }\end{array}$ & $11,247 / 1,844$ & $72.5 ;$ na & Dementia & Sleep characteristics \\
\hline Niu, 2016 & China & $1 y(\max )$ & $1,010 / 161$ & $69.8 ; 65 \%$ & Cognitive decline & Sleep characteristics \\
\hline Ding, 2016 & USA/Canada & 5.7y (mean) & $7,547 / 310$ & $67.5 ; 0 \%$ & Dementia & Sleep apnoea \\
\hline Diem, 2016 & USA & 4.9y (mean) & $\begin{array}{l}\text { 1,245/290 MCl and } 183 \\
\text { dementia }\end{array}$ & $82.6 ; 100 \%$ & Dementia or $\mathrm{MCl}$ & Sleep characteristics \\
\hline Yaffe, 2011 & USA & $4.7 y$ (mean) & 298/60 MCl and 47 dementia & $82.3 ; 100 \%$ & Dementia or $\mathrm{MCl}$ & Sleep characteristics \\
\hline Chen, 2016 & USA & 7.3-7.7 (mean) & $7,444 / 802$ & $70.1 ; 100 \%$ & Cognitive decline & Sleep duration \\
\hline Yaffe, 2015 & USA & $8 y(\max )$ & $\begin{array}{l}\text { 179,738/21,784 dementia } \\
(4,107 \mathrm{AD} \text { and } 2,715 \mathrm{VD})\end{array}$ & $66.9-68.5 ; 0 \%$ & $\begin{array}{l}\text { Dementia and its } \\
\text { subtypes }\end{array}$ & Series of sleep problems \\
\hline Tsapanou, 2015 & USA & $3 y(\max )$ & $1,041 / 78$ & $79.3 ; 69.8 \%$ & Dementia & Series of sleep problems \\
\hline Martin, 2015 & France & $7.8 y$ (mean) & 559/na & $67.0 ; 60.3 \%$ & Attentional decline & SBD \\
\hline Lin, 2015 & China-Taiwan & $5 y(\max )$ & $3,020 / 166$ & Mixed; $43.7 \%$ & $\begin{array}{l}\text { Dementia and its } \\
\text { subtypes }\end{array}$ & $\begin{array}{l}\text { Sleep-related movement } \\
\text { disorders }\end{array}$ \\
\hline Chiu, 2015 & China-Taiwan & $7 y(\max )$ & $5,960 / 88$ & Mixed; $49 \%$ & Dementia & Insomnia \\
\hline Song, 2015 & USA & $3.4 y$ (mean) & $2,601 / 484$ & $76.0 ; 0 \%$ & Cognitive decline & Sleep stage duration \\
\hline Blackwell, 2015 & USA & $3.4 y$ (mean) & $2,636 / 484$ & $76.0 ; 0 \%$ & Cognitive decline & SBD \\
\hline Blackwell, 2014 & USA & $3.4 y$ (mean) & $2,822 / 484$ & $76.0 ; 0 \%$ & Cognitive decline & Sleep characteristics \\
\hline Benedict, 2015 & Sweden & $40 y(\max )$ & $\begin{array}{l}\text { 1,574/270 dementia ( } 119 \\
\text { pure } A D \text { and } 61 \mathrm{VD})\end{array}$ & $49.6 ; 0 \%$ & $\begin{array}{l}\text { Dementia and its } \\
\text { subtypes }\end{array}$ & Sleep disturbances \\
\hline Hahn, 2014 & Sweden & $9 y(\max )$ & 214/61 dementia (47 AD) & $83.4 ; 80.4 \%$ & $\begin{array}{l}\text { Dementia and its } \\
\text { subtypes }\end{array}$ & Sleep pattern change \\
\hline Virta, 2013 & Finland & 22.6y (mean) & $1,326 / 179$ & Midlife; $47.9 \%$ & $A D$ & Sleep characteristics \\
\hline Sterniczuk, 2013 & 12 countries in Europe & 4.3y (mean) & $17,656 / 300$ & $63.9 ; 55.5 \%$ & Dementia & Sleep characteristics \\
\hline Peters, 2013 & USA & $3.3 y$ (mean) & 230/61 dementia (47 AD) & $81.9 ; 50 \%$ & $\begin{array}{l}\text { Dementia and its } \\
\text { subtypes }\end{array}$ & Nighttime behaviours \\
\hline Lim, 2013 & USA & $6 y(\max ) ; 3.3 y$ (mean) & $737 / 97$ & $81.6 ; 76 \%$ & $A D$ & Sleep fragmentation \\
\hline Benito-Leon, 2013 & Spain & $3.4 y$ (mean) & $2,715 / \mathrm{na}$ & $72.9 ; 57 \%$ & Cognitive decline & Sleep duration \\
\hline Benito-León, 2009 & Spain & $3.2 y$ (median) & $3,286 / 140$ & $72.9 ; 57 \%$ & $\begin{array}{l}\text { Dementia and its } \\
\text { subtypes }\end{array}$ & Sleep duration \\
\hline
\end{tabular}


Cognitive neurology

\begin{tabular}{|c|c|c|c|c|c|c|}
\hline First author, year * & $\begin{array}{l}\text { Design, cohort name, } \\
\text { country }\end{array}$ & Observation & Sample for analysis/cases & Mean age; female & Outcome & $\begin{array}{l}\text { Type of sleep } \\
\text { disorders }\end{array}$ \\
\hline Potvin, 2012 & Canada & $1 y(\max )$ & $1,664 / 68$ women +37 men & $74.4 ; 69.7 \%$ & Cognitive decline & Sleep characteristics \\
\hline Keage, 2012 & Canada & $10 y(\max )$ & $604 / \mathrm{na}$ & $75 ; 53 \%$ & Cognitive decline & Sleep characteristics \\
\hline Jaussent, 2012 & France & $8 y(\max )$ & $4,898 / 697$ & Range $=65-85 ; 57 \%$ & Cognitive decline & Sleep characteristics \\
\hline Boot, 2012 & USA & 3.8y (median) & $\begin{array}{l}651 \text { at least one follow- } \\
\text { up/104 }\end{array}$ & $77 ; 30 \%$ & $\mathrm{MCl}$ & RBD \\
\hline Osorio, 2011 & USA & 7.7y (mean) & $346 / 25$ & Range: $24-96 ; 64 \%$ & $A D$ & Insomnia \\
\hline Elwood, 2011 & UK & 10y (max) & 1,225/49 AD and $44 \mathrm{VD}$ & $61.5 ; 0 \%$ & $A D$ and $V D$ & Sleep characteristics \\
\hline Lobo, 2008 & Spain & $2 y(\max )$ & $\begin{array}{l}\text { 3,244/82 dementia (47 AD } \\
\text { and } 208 \mathrm{MCl} \text { ) }\end{array}$ & $73.6 ; 55.2 \%$ & Dementia; AD; $\mathrm{MCl}$ & Sleep problems \\
\hline Tworoger, 2006 & USA & $1.8 y$ (mean) & $1,844 / \mathrm{na}$ & $74 ; 100 \%$ & Cognitive decline & Sleep characteristics \\
\hline \multirow[t]{2}{*}{ Foley, 2001} & USA & $3 y(\max )$ & $2,346 / 191$ & $76.6 ; 0 \%$ & Dementia & Sleep characteristics \\
\hline & & & $2,242 / 482$ & & Cognitive decline & \\
\hline \multirow[t]{2}{*}{ Cricco, 2001} & USA & 3y (max) & $2,429 / 619$ & $72 ;$ na & Cognitive decline & Insomnia \\
\hline & & & $4,015 / 1076$ & & & \\
\hline Quesnot, 1999 & France & $4 y(\max )$ & $1,389 / 184$ & Range: $59-71 ; 58.7 \%$ & Cognitive decline & $\begin{array}{l}\text { Snoring and excessive } \\
\text { daytime sleepiness }\end{array}$ \\
\hline
\end{tabular}

${ }^{*}$ The full reference list can be found in online supplementary appendix 3.

AD, Alzheimer's disease; LCI, Lower Confidence Interval; MCI, Mild Cognitive Impairment; na, Not Applicable; PSQI, Pittsburgh Sleep Quality Index; RBD, Rapid Eye Movement Sleep Behavioural Disorder; REM, Rapid Eye Movement; REMR, Robust Error Meta-regression; SBD, Sleep Breathing Disorders; UCI, Upper Confidence Interval; VD, Vascular Dementia; $y$, year.

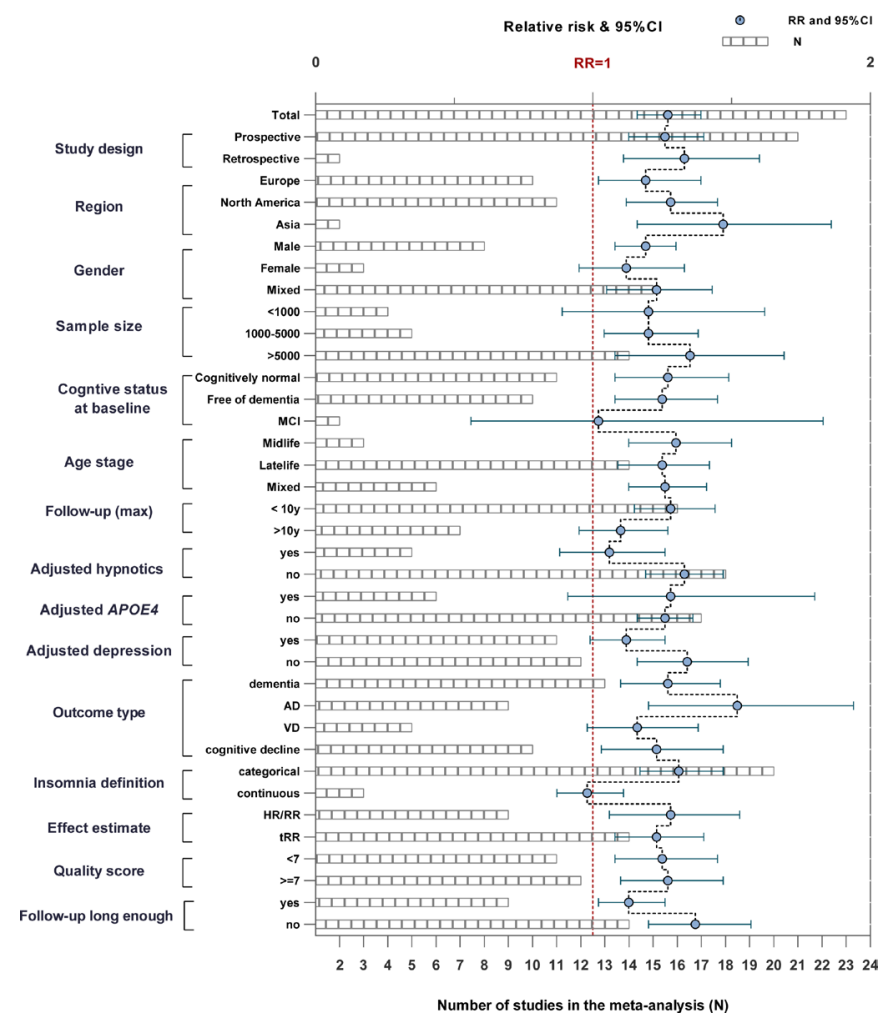

Figure 2 Association of insomnia with risk of cognitive disorders. Subgroup analyses indicated that the significance of the primary result was not altered by study design, region, gender, cognitive status at baseline, age stage, effect estimate, quality score or $A D$ as an outcome. However, the pooled results tend to be non-significant in strata of small-sample studies, studies with longer follow-up, studies adjusted for more covariates, studies with VD as an outcome or insomnia defined as a continuous variable. $A D$, Alzheimer's disease; $\mathrm{MCl}$, mild cognitive impairment; $\mathrm{RR}$, relative risk. studies $^{25-27}$ that used continuous variables and one repetitive sample ${ }^{28}$ were excluded, leaving 17 for meta-analyses (including 12 for nocturnal and five for total daily duration). All studies are prospective cohort studies with populations from North America (two in Canada and seven in America), Europe (one in Finland, one in Spain and two in Sweden) and East Asia (one in China, one in South Korea and two in Japan). The mean age varied from 51 to 83 years old and the mean follow-up varied from 1 to 22.6 years. The average study quality is moderate (median score $=7$ ).

A nonlinear trend was revealed for the relationship between sleep duration and cognitive disorders. Subgroup analyses indicated that the trend persists for $\mathrm{AD}$, but not for VD (table 2). The dose-response analyses revealed significant nonlinear associations between sleep duration and risks of cognitive disorders $(\mathrm{p}=0.0003$ for nocturnal and $\mathrm{p}=0.017$ for total daily duration) or $\mathrm{AD}$ ( $\mathrm{p}=0.018$ for nocturnal) (figure 5). Specifically, as for the sleep duration at night, the optimal duration was found to be roughly 5.6-6hours for lower risk of cognitive disorder (figure 5A) and 5.6-7 hours for lower risk of $\mathrm{AD}$ (figure 5B). The risk of cognitive disorder (figure $5 \mathrm{~A}$ ) or $\mathrm{AD}$ (figure $5 \mathrm{~B}$ ) will be significantly elevated when the nocturnal sleep duration is over 10 hours or less than 4 hours. Similar results were obtained for the associations with total daily sleep duration: the protective window was situated between 5.6 and 9 hours for lower risk of cognitive disorders (figure $5 \mathrm{C}$ ). The nonlinearity in the relationship between daily duration and $\mathrm{AD}$ (figure 5D) or VD showed borderline significance $(p=0.069)$, possibly because of the limited number of included studies.

\section{Change of sleep characteristics and cognitive disorders}

Moreover, several studies reported associations between cognitive decline and change of sleep pattern, ${ }^{29}$ that is, change in sleep characteristics, including duration, ${ }^{12}{ }^{30-32}$ depth, ${ }^{32}$ latency ${ }^{30}$ and variability. ${ }^{33}$ Meta-analyses of change in sleep duration found that increased duration rather than reduced duration could 


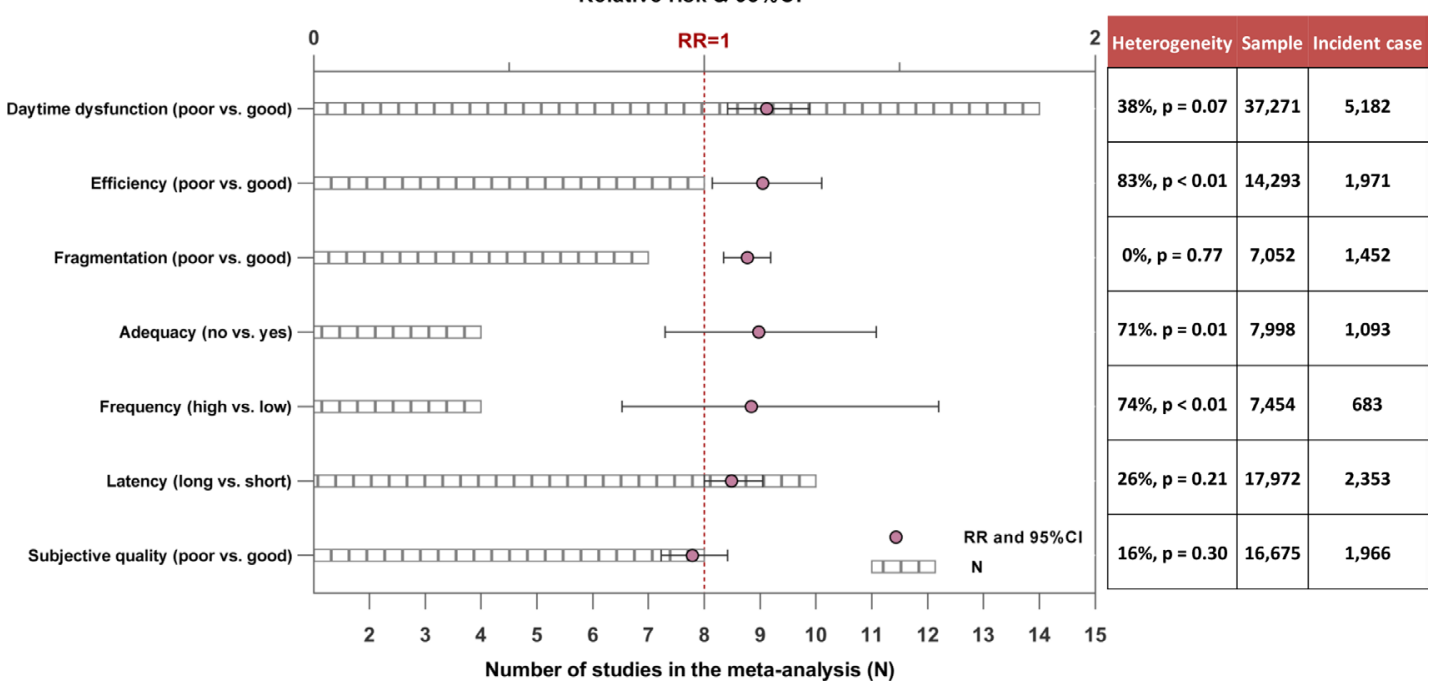

Figure 3 Associations between components of insomnia and cognitive disorders. Four insomnia components were associated with 7\%-16\% increased risk of cognitive disorders, including daytime dysfunction, inefficiency, fragmentation and latency. Adequacy, high frequency of insomnia and subjective quality showed no significant associations in primary analyses. RR, relative risk.

significantly elevate the risk of cognitive disorders in nondemented elderly (online supplementary appendix 5).

\section{Rating of evidence levels}

As for the levels of credibility, three meta-analyses (insomnia, fragmentation and subjective quality) were rated at a moderateto-high level ( $G$ and $A+$ level) and four (daytime dysfunction, latency, RBD and TIB) were rated at a moderate level (A- level). In addition, we found that eight meta-analyses were rated at $S$ level (frequency, apnoea, snoring and napping) or P level (efficiency, adequacy, SBD and RLS). Poor generalisability, follow-up inadequacy and attrition are major sources of bias (figure 6).

\section{DISCUSSION}

We found evidence supporting 10 types of self-reported sleep conditions or parameters, including six (insomnia, fragmentation, daytime dysfunction, prolonged latency, RBD and excessive TIB) with moderate-to-high levels of evidence and four (apnoea, no habitual napping, inefficiency, increased sleep duration) with low levels of evidence, as predictors of higher risk of cognitive disorders in non-demented adults. The quantitative metaanalysis indicated that either insufficient $(<4$ hours per night or total daily) or excessive ( $>10$ hours per night and $>12.5$ hours for total daily) sleep duration could elevate risk of all-cause cognitive disorders or AD dementia.

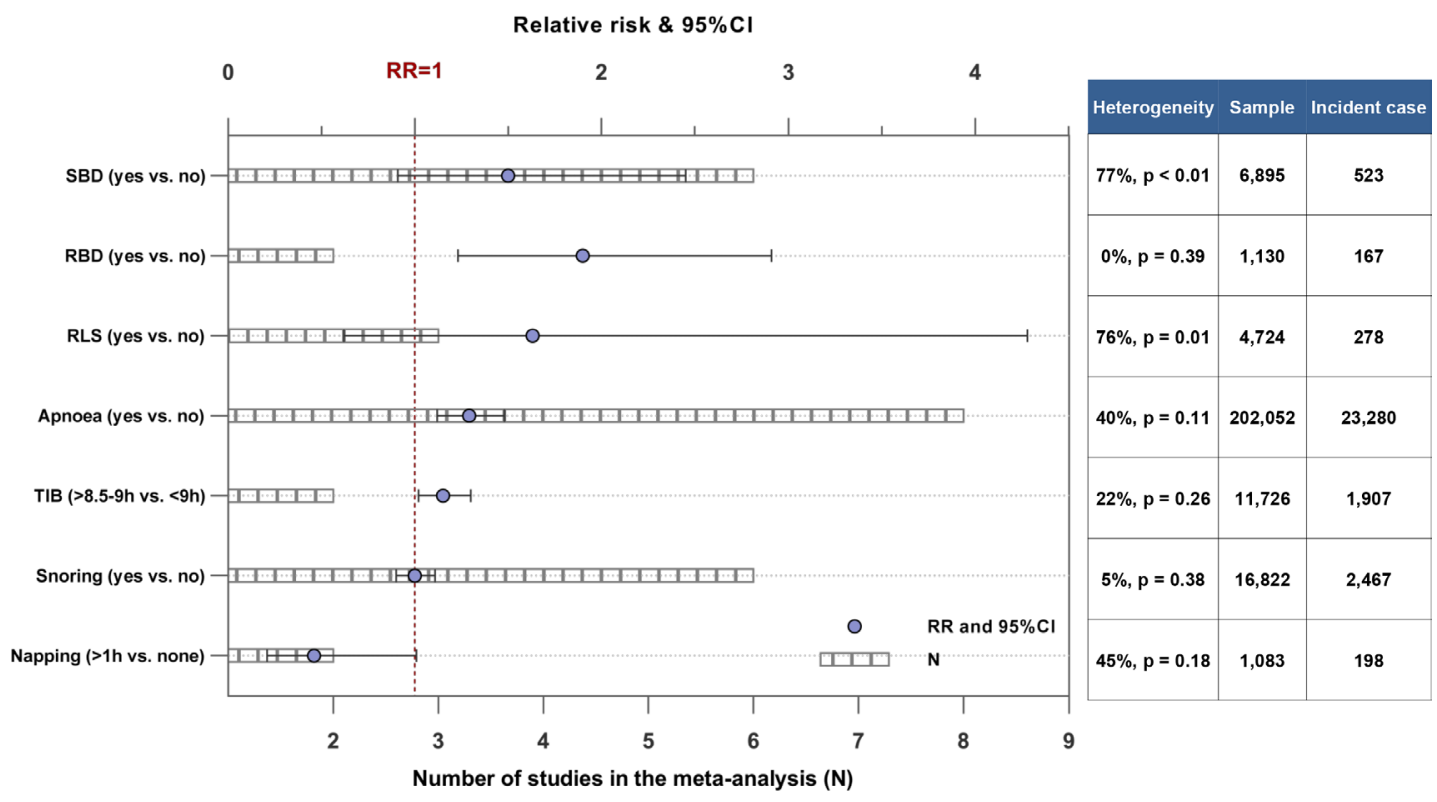

Figure 4 Associations of sleep-related problems with cognitive disorders. Four types of sleep-related problems showed significant associations with risk of cognitive disorders, including rapid eye movement sleep behavioural disorder (RBD), apnoea, longer time in bed (TIB) and habitual napping. We identified no significant association with restless leg syndrome (RLS) or snoring. RR, relative risk; SBD, sleep behavioural disturbance. 
Cognitive neurology

\begin{tabular}{|c|c|c|c|c|c|c|c|}
\hline Outcome & Exposure level & Definition & N & Sample size (case) & Pooled results & $I^{2}$ ( $p$ value) & P for publication bias \\
\hline \multirow{4}{*}{$\begin{array}{l}\text { Dementia or } \\
\text { cognitive decline }\end{array}$} & Highest vs ref & Nocturnal & 13 & $53,014(6,892)$ & $1.41(1.17-1.70)$ & $69 \%(<0.01)$ & 0.4394 \\
\hline & Lowest vs ref & & & & $1.18(1.01-1.37)$ & $57 \%(<0.01)$ & 0.1033 \\
\hline & Highest vs ref & Daily & 4 & $6,925(854)$ & $1.73(1.30-2.31)$ & $9.6 \%(0.345)$ & NA \\
\hline & Lowest vs ref & & & & $1.83(1.38-2.43)$ & $0 \%(0.563)$ & NA \\
\hline \multirow[t]{4}{*}{$A D$} & Highest vs ref & Nocturnal & 3 & $32,555(4,115)$ & $1.57(1.33-1.85)$ & $0 \%(0.90)$ & NA \\
\hline & Lowest vs ref & & & & $1.02(0.76-1.36)$ & $54 \%(0.11)$ & NA \\
\hline & Highest vs ref & Daily & 2 & 4,783 (296) & $2.47(1.55-3.93)$ & $0 \%(0.91)$ & NA \\
\hline & Lowest vs ref & & & & $2.27(1.17-4.38)$ & $0 \%(0.89)$ & NA \\
\hline \multirow[t]{2}{*}{ VD } & Highest vs ref & Daily & 2 & $4,783(117)$ & $2.20(1.12-4.32)$ & $0 \%(0.60)$ & NA \\
\hline & Lowest vs ref & & & & $2.33(0.72-7.49)$ & $45 \%(0.18)$ & NA \\
\hline
\end{tabular}

AD, Alzheimer's disease; NA, not applicable.

The high heterogeneity observed for association between insomnia and cognitive disorders might be explained by the varying definitions of insomnia. The heterogeneity levels were significantly lowered when the analyses were restricted to its component domains (such as daytime dysfunction, fragmentation, latency and subjective quality). As for the subtypes of cognitive disorders, insomnia tends to influence risk of AD but not VD. However, the associations between $\mathrm{AD}$ and insomnia components have been scarcely reported ${ }^{34}$ and should be further investigated in the future (online supplementary appendix 4). Interestingly, the associations became non-significant when specific factors (hypnotics, APOE4 status and depression at baseline) were included as covariates, suggesting the potential existence of stratified or mediating effects. The mechanisms underpinning the association with insomnia might be related to inflammation. ${ }^{35}$

We found that apnoea is another important sleep-related risk factor for all-cause cognitive disorders. Obstructive sleep apnoea has been previously linked with poorer performance in multiple cognitive domains among non-demented individuals, including attention, executive functioning, visuospatial and constructional abilities and psychomotor speed. ${ }^{36}$ The mechanism might be related to long-term cerebral hypoxia and hypometabolism, which might contribute to loss of regional cortex and white matter hyperintensities in the hippocampus and cingulate cortex. $^{35}$

A U-shaped relationship was confirmed between self-reported nocturnal or total daily sleep duration and all-cause cognitive
A

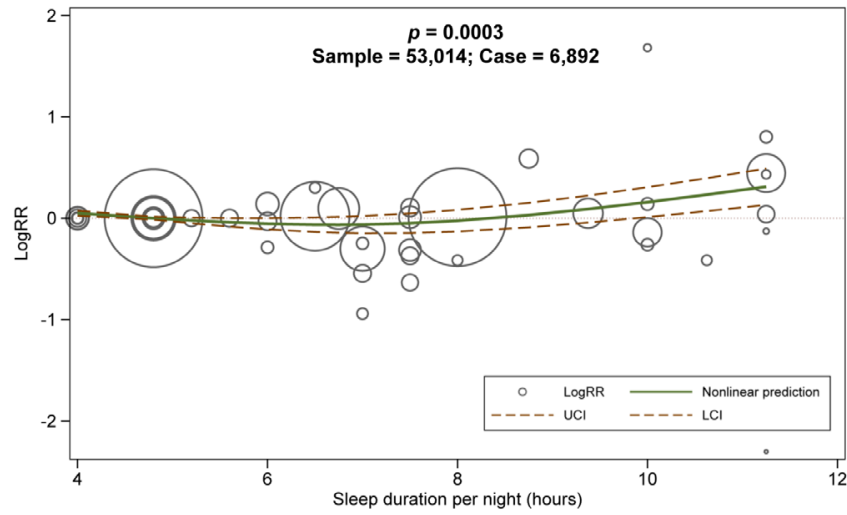

C

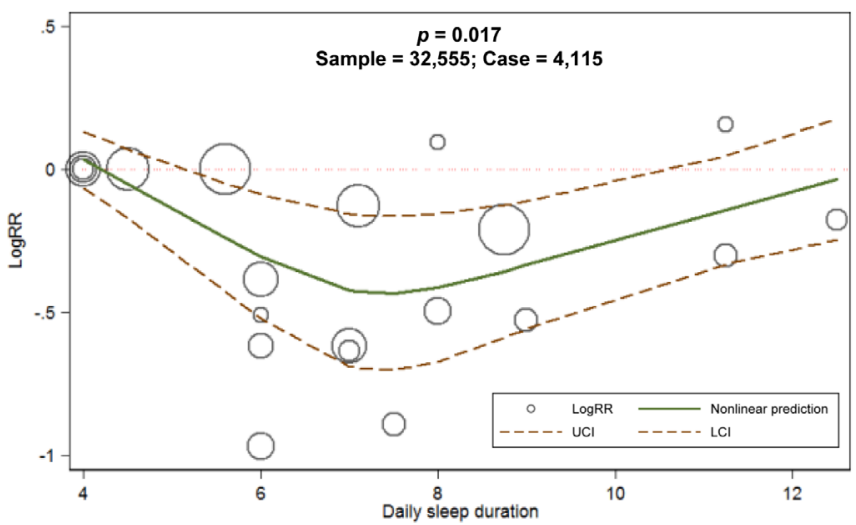

B

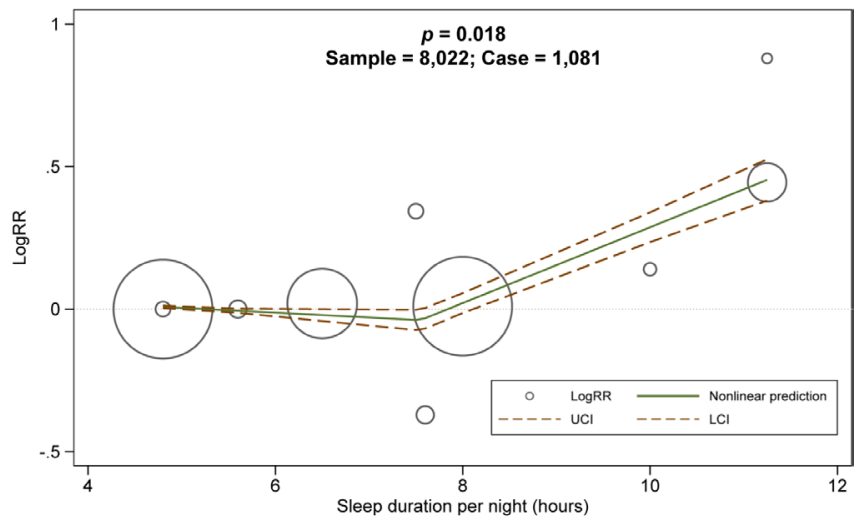

D

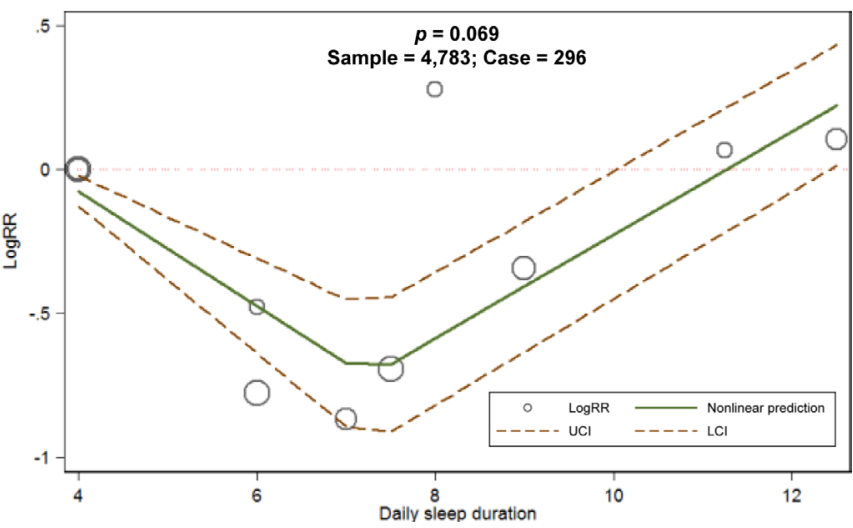

Figure 5 Dose-response relationships between sleep duration and cognitive disorders or AD. The dose-response analyses revealed significantly nonlinear associations between nocturnal or total daily sleep duration and risk of cognitive disorders ( $A$ and $C$ ) or $A D(B$ and $D)$. AD, Alzheimer's disease; RR, relative risk. 


\begin{tabular}{|c|c|c|c|c|c|c|c|}
\hline \multicolumn{2}{|c|}{$\begin{array}{c}\text { Characteristics or types of sleep } \\
\text { problem }\end{array}$} & \begin{tabular}{|c|} 
Risk \\
of bias
\end{tabular} & Inconsistency & Imprecision & \begin{tabular}{|c} 
Publication \\
bias
\end{tabular} & $\begin{array}{c}\text { Evidence } \\
\text { grade }\end{array}$ & WQS \\
\hline \multicolumn{2}{|c|}{ Insomnia } & $0^{*}$ & $0^{*}$ & -1 & 0 & $A+$ & 6.50 \\
\hline \multirow{7}{*}{$\begin{array}{l}\text { Components } \\
\text { of insomnia }\end{array}$} & Daytime dysfunction & -1 & 0 & -1 & 0 & A - & 6.78 \\
\hline & Efficiency & -1 & -2 & -1 & na & $\mathrm{P}$ & 6.91 \\
\hline & Fragmentation & -1 & 0 & 0 & na & $A+$ & 6.83 \\
\hline & Adequacy & -1 & -2 & -1 & na & $P$ & 6.76 \\
\hline & Frequency & 0 & -2 & -1 & na & $S$ & 7.33 \\
\hline & Latency & -1 & 0 & -1 & 0 & A - & 6.95 \\
\hline & Subjective quality & 0 & 0 & 0 & na & $G$ & 7.05 \\
\hline \multirow{7}{*}{$\begin{array}{c}\text { Other types } \\
\text { of sleep } \\
\text { problems }\end{array}$} & SBD & -1 & -2 & -1 & na & $\mathrm{P}$ & 6.48 \\
\hline & RBD & -1 & 0 & -1 & na & A - & 7.11 \\
\hline & RLS & -2 & -2 & -2 & na & $P$ & 6.02 \\
\hline & Apnoea & -2 & 0 & -1 & na & $S$ & 6.24 \\
\hline & TIB & -1 & 0 & -1 & na & A - & 6.50 \\
\hline & Snoring & -2 & 0 & 0 & na & $S$ & 6.27 \\
\hline & Napping & -1 & -1 & -1 & na & $S$ & 6.81 \\
\hline
\end{tabular}

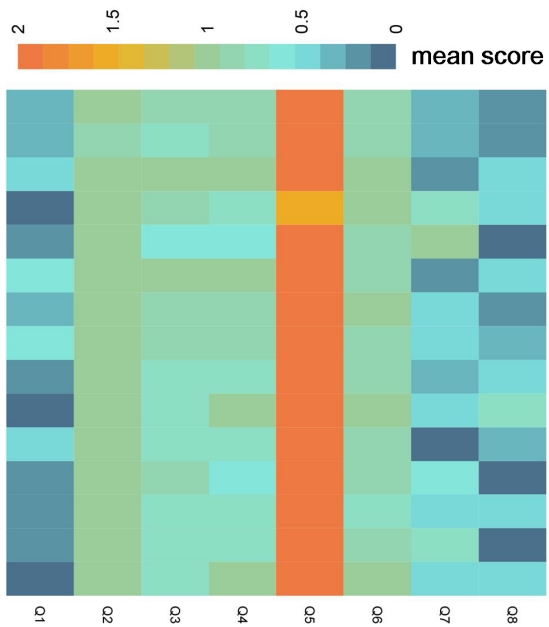

Figure 6 Evidence rating for meta-analyses results. Three meta-analyses (insomnia, fragmentation and subjective quality) were rated at a moderate-tohigh level and four (daytime dysfunction, latency, RBD and TIB) were rated at a moderate level. In addition, we found that eight meta-analyses were rated at $\mathrm{S}$ level or P level. Poor generalisability, follow-up inadequacy and large attrition are major sources of bias. RBD, rapid eye movement sleep behavioural disorder; RLS, restless leg syndrome; SBD, sleep behavioural disturbance; TIB, time in bed.

disorders or $\mathrm{AD}$, which is consistent with previous findings. ${ }^{37}$ One meta-analysis ${ }^{37}$ incorporating five cohorts and four crosssectional studies suggested the optimal sleep duration approximate 7 hours, which is slightly different from ours (6.3 hours at night and 7.3 hours for total daily sleep duration). Because we included 17 cohort studies for dose-response analyses and conducted separate analyses for the nocturnal and total daily sleep durations. The underlying mechanisms might be explained by that shorter or longer sleep duration contributes to faster atrophy in frontotemporal region, ${ }^{38}$ ventricular enlargement ${ }^{39}$ and hippocampal degeneration. ${ }^{40}$ Another possible explanation is that those who already have somedegeneration, or other comorbidities and medication use tend to sleep longer.

Compared with previous meta-analyses (online supplemenatry appendix 6), the present study had several significant advantages: (1) only longitudinal cohort studies were included, (2) the sleep disorder spectrum was fully covered and separately explored for each domain, (3) as an updated systematic review, we included 33 other literatures compared with the latest systematic review, ${ }^{13}$ (4) the dose-response relationship for sleep duration was explored, (5) stratified analyses according to different types of sleep or cognitive disorders were conducted to lower the heterogeneity of the pooled results and (6) evidence robustness was rated.

Several limitations exist. First, the associations identified by the analyses based on observational cohort studies were not equal to causal relationships. Randomised controlled trials are warranted in the future to test the roles of sleep management in preventing cognitive decline or dementia. Second, we did not explore the association of hypnotics with risk of cognitive disorders in the present study though they were closely linked with sleep. Instead, we conducted subgroup analyses according to whether sleep medication was included as a covariate. Third, the associations with dementia subtype (AD or VD) were not thoroughly investigated due to the limited evidence. Fourth, the influences of important confounders, such as cardiovascular diseases are not fully explored,

In conclusion, the findings of the our study provided varying levels of evidence that 10 types of sleep-related exposures were linked to increased risk of all-cause cognitive decline or dementia. Future studies are warranted to confirm the associations with $\mathrm{AD}$ and to examine the roles of sleep management in benefiting cognition and lowering risk of dementia.

\section{TwitterWei Xu@na}

Contributors WX: conceptualisation and design of the study, collection and analysis of the data, drafting and revision of the manuscript, and prepared all the figures. C-CT: collection and analysis of the data, and revision of the manuscript. J-JZ, $X-P C$ and LT: revision of the manuscript.

Funding The authors have not declared a specific grant for this research from any funding agency in the public, commercial or not-for-profit sectors.

Competing interests None declared.

Patient consent for publication Not required.

Provenance and peer review Not commissioned; externally peer reviewed. Data availability statement Data are available upon reasonable request.

Open access This is an open access article distributed in accordance with the Creative Commons Attribution Non Commercial (CC BY-NC 4.0) license, which permits others to distribute, remix, adapt, build upon this work non-commercially, and license their derivative works on different terms, provided the original work is properly cited, appropriate credit is given, any changes made indicated, and the use is non-commercial. See: http://creativecommons.org/licenses/by-nc/4.0/.

\section{ORCID iD}

Wei Xu http://orcid.org/0000-0002-3310-5875

\section{REFERENCES}

1 Branger $P$, Mézenge $F$, André $C$, et al. Difficulties falling asleep is associated with higher Abeta burden in healthy adults. Alzheimers Dement 2015;11:P390-1.

2 Lucey BP, Hicks TJ, McLeland JS, et al. Effect of sleep on overnight cerebrospinal fluid amyloid $\beta$ kinetics. Ann Neurol 2018;83:197-204.

3 Lucey BP, Hicks TJ, McLeland JS, et al. Sleep loss increases risk of Alzheimer's disease by increasing CNS ab production. Ann Neurol 2017;82:S53-4.

4 Carvalho DZ, St Louis EK, Knopman DS, et al. Association of excessive daytime sleepiness with longitudinal $\beta$-amyloid accumulation in elderly persons without dementia. JAMA Neurol 2018;75:672-80.

5 Shim A, Hogan M, Halldin K, et al. Sleep disordered breathing, ApoE4 and $\beta$-amyloid deposition in cognitively normal elderly. Alzheimers Dement 2017;13:P1124-5.

6 Díaz M, Pulópulos M, Baquero M, et al. Sleep disorders in mild cognitive impairment. Alzheimers Dement 2017;13

7 Hooghiemstra AM, Visser PJ, Slot RER, et al. Alzheimer's disease patients with osas history have higher csf tau levels. Alzheimers Dement 2016;12.

8 Brown BM, Rainey-Smith SR, Villemagne VL, et al. Investigating the synergistic relationship between sleep quality, physical activity, and levels of brain beta-amyloid. Alzheimers Dement 2015;11:P451-254 
9 Benedict C, Byberg L, Cedernaes J, et al. Self-Reported sleep disturbance is associated with Alzheimer's disease risk in men. Alzheimer's \& Dementia 2015;11:1090-7.

10 Lutsey PL, Misialek JR, Mosley TH, et al. Sleep characteristics and risk of dementia and Alzheimer's disease: the Atherosclerosis risk in Communities study. Alzheimers Dement 2018;14:157-66.

11 Yaffe K, Laffan AM, Harrison SL, et al. Sleep-Disordered breathing, hypoxia, and risk of mild cognitive impairment and dementia in older women. JAMA 2011;306:613-9.

12 Westwood AJ, Beiser A, Jain N, et al. Prolonged sleep duration as a marker of early neurodegeneration predicting incident dementia. Neurology 2017;88:1172-9.

13 Shi L, Chen S-J, Ma M-Y, et al. Sleep disturbances increase the risk of dementia: a systematic review and meta-analysis. Sleep Med Rev 2018;40:4-16.

14 Moher D, Liberati A, Tetzlaff J, et al. Preferred reporting items for systematic reviews and meta-analyses: the PRISMA statement. Int J Surg 2010;8:336-41.

15 Stroup DF, Berlin JA, Morton SC, et al. Meta-Analysis of observational studies in epidemiology: a proposal for reporting. meta-analysis of observational studies in epidemiology (moose) group. JAMA 2000;283:2008-12.

16 Higgins JPT, Thompson SG, Deeks JJ, et al. Measuring inconsistency in meta-analyses. BMJ 2003;327:557-60.

17 Grant RL. Converting an odds ratio to a range of plausible relative risks for better communication of research findings. BMJ 2014;348:f7450.

18 Riley RD, Higgins JPT, Deeks JJ. Interpretation of random effects meta-analyses. $B M J$ 2011;342:d549.

19 Hedges LV, Tipton E, Johnson MC. Robust variance estimation in meta-regression with dependent effect size estimates. Research Synthesis Methods 2010;1:39-65.

$20 \mathrm{XU}$ C, Doi SAR. The robust error meta-regression method for dose-response metaanalysis. Int J Evid Based Healthc 2018;16:138-44.

21 Orsini N. From floated to conventional confidence intervals for the relative risks based on published dose-response data. Comput Methods Programs Biomed 2010;98:90-3.

22 Burke SL, Cadet T, Alcide A, et al. Psychosocial risk factors and Alzheimer's disease: the associative effect of depression, sleep disturbance, and anxiety. Aging Ment Health 2018;22:1577-84

23 Lobo A, LóPez-Antón R, De-La-CÁmara C, et al. Non-cognitive psychopathological symptoms associated with incident mild cognitive impairment and dementia, alzheimer's type. Neurotox Res 2008;14:263-72

24 Jaussent I, Bouyer J, Ancelin M-L, et al. Excessive sleepiness is predictive of cognitive decline in the elderly. Sleep 2012:35:1201-7.
25 Lysen TS, Wolters FJ, Luik Al, et al. Subjective sleep quality is not associated with incident dementia: the Rotterdam study. J Alzheimers Dis 2018;64:239-47.

26 Gabelle A, Gutierrez L-A, Jaussent I, et al. Excessive sleepiness and longer nighttime in bed increase the risk of cognitive decline in frail elderly subjects: the MAPT-sleep study. Front Aging Neurosci 2017;9

27 Tsapanou A, Gu Y, Manly J, et al. Daytime sleepiness and sleep inadequacy as risk factors for dementia. Dement Geriatr Cogn Dis Extra 2015;5:286-95.

28 Benito-León J, Louis ED, Bermejo-Pareja F. Cognitive decline in short and long sleepers: a prospective population-based study (NEDICES). J Psychiatr Res 2013;47:1998-2003.

29 Sterniczuk R, Theou O, Rusak B, et al. Sleep disturbance is associated with incident dementia and mortality. Current Alzheimer research 2013;10:767-75.

30 Suh SW, Han JW, Lee JR, et al. Sleep and cognitive decline: a prospective nondemented elderly cohort study. Ann Neurol 2018;83:472-82.

31 Lu Y, Sugawara Y, Zhang $S$, et al. Changes in sleep duration and the risk of incident dementia in the elderly Japanese: the Ohsaki cohort 2006 study. Sleep 2018;41.

32 Hahn EA, Wang H-X, Andel R, et al. A change in sleep pattern may predict Alzheimer disease. Am J Geriatr Psychiatry 2014;22:1262-71.

33 Diem SJ, Blackwell TL, Stone KL, et al. Measures of sleep-wake patterns and risk of mild cognitive impairment or dementia in older women. Am J Geriatr Psychiatry 2016;24:248-58.

34 Pase MP, Himali JJ, Grima NA, et al. Sleep architecture and the risk of incident dementia in the community. Neurology 2017;89:1244-50.

35 Irwin MR, Vitiello MV. Implications of sleep disturbance and inflammation for Alzheimer's disease dementia. Lancet Neurol 2019;18:296-306.

36 Wallace A, Bucks RS. Memory and obstructive sleep apnea: a meta-analysis. Sleep 2013;36:203-20

37 Liang Y, Qu L-B, Liu H. Non-Linear associations between sleep duration and the risks of mild cognitive impairment/dementia and cognitive decline: a dose-response meta-analysis of observational studies. Aging Clin Exp Res 2019;31:309-20.

38 Spira AP, Gonzalez CE, Venkatraman VK, et al. Sleep duration and subsequent cortical thinning in cognitively normal older adults. Sleep 2016;39:1121-8.

$39 \mathrm{JC}$ L, Loh KK, Zheng H, et al. Sleep duration and age-related changes in brain structure and cognitive performance. Sleep 2014:37:1171-8.

40 Tononi G, Cirelli C. Sleep and the price of plasticity: from synaptic and cellular homeostasis to memory consolidation and integration. Neuron 2014;81:12-34. 